\title{
TOMOGRAFIA DE IMPULSO PARA AVALIAÇÃO DO INTERIOR DO LENHO DE ÁRVORES
}

Luciana Cavalcante Pereira ${ }^{1}$, Demóstenes Ferreira da Silva Filho², Mário Tomazello Filho ${ }^{3}$, Hilton Tadeu Zarate do Couto ${ }^{4}$, José Mauro M. Á. Paz Moreira ${ }^{5}$, Jefferson Lordello Polizel ${ }^{6}$

\section{RESUMO}

As árvores só podem persistir se mecanicamente confiáveis e seguras. Problemas no tronco aumentam o risco de queda e potencializam acidentes. Antes do uso da tomografia, era preciso derrubar as árvores para visualizar seu interior. A tomografia de impulso é uma técnica que vem sendo desenvolvida para avaliação do interior do lenho. Baseia-se na cronometragem, por meio de sensores, de ondas mecânicas que atravessam a madeira e no cálculo da velocidade dessas ondas com auxílio de computador. A representação da distribuição das velocidades de onda ao longo de seção transversal do tronco origina a imagem tomográfica. Este estudo pretendeu verificar a qualidade das informações fornecidas pela tomografia de impulso. Aferiu-se os dados produzidos pela tomografia de impulso comparando-os aos dados produzidos pela densitometria de raio $\mathrm{X}$, técnica confiável de obtenção da densidade aparente de amostras diametrais do lenho. Foram amostrados toretes de árvores de três espécies para aplicação do tomógrafo de impulso em seção transversal dos mesmos. Uma amostra diametral de cada seção transversal tomografada foi submetida à densitometria. Observou-se correlação dos dados de velocidade de onda mecânica e de densidade aparente. A tomografia de impulso produziu dados que representaram corretamente o interior das amostras de lenho estudadas.

Palavras-chave: risco de queda, árvores maduras, avaliação não-destrutiva, densitometria de raio $X$, tomografia de árvore.

\footnotetext{
${ }^{1}$ Graduanda em Engenharia Agronômica, USP/ESALQ, Piracicaba/ SP, e-mail: Icavalp@esalq.usp.br

2 Engenheiro Agrônomo, Professor, USP/ESALQ, Piracicaba/SP, e-mail: dfsilva@esalq.usp.br

${ }^{3}$ Engenheiro Agrônomo, Professor, USP/ESALQ, Piracicaba/SP, e-mail: mtomazel@esalq.usp.br

${ }^{4}$ Engenheiro Agrônomo, Professor, USP/ESALQ, Piracicaba/SP, e-mail: htzcouto@esalq.usp.br

${ }^{5}$ Doutorando em Economia Aplicada, USP/ESALQ, Piracicaba/SP, e-mail: jmoreira@esalq.usp.br

${ }^{6}$ Mestrando em Geografia Física , USP/FFLCH, São Paulo/SP, e-mail: jlpolize@esalq.usp.br
} 


\title{
IMPULSE TOMOGRAPHY FOR EVALUATION OF THE INTERIOR OF THE LOG OF TREES
}

\begin{abstract}
The trees can only persist if mechanically reliable and safe. Problems in the trunk increase the fall risk and propitiate accidents. Before the use of tomography, it was necessary to cut the tree to visualize its interior. The impulse tomography is a technique that has been developed for the interior of log trees evaluation. Using sensors, it is possible to chronometer mechanical waves that cross the wood and using a computer, its speed is calculated. The representation of the distribution of the wave speeds through cross section of the trunk originates the tomographic image. This study intended to verify the quality of the information supplied by the impulse tomography. The data produced by the impulse tomography were compared with the data produced by the X-ray densitometry, a reliable technique to obtain the apparent density of diametrical samples of the log. Samples of trunks were gotten of three trees species for the impulse tomography application in their cross section. A diametrical sample of each tomographed cross section was submitted to the X-ray densitometry. Correlation between the mechanic waves speed data and the apparent density data was observed. The impulse tomography produced data that represented correctly the interior of the studied log samples.
\end{abstract}

Key words: fall risk tree, mature trees, non-destructive evaluation, X-ray densitometry, tree tomography. 


\section{INTRODUÇÃO}

Para persistir e sobreviver em qualquer ambiente, uma árvore deve ser mecanicamente confiável e possuir razoável fator da segurança. Problemas no tronco atribuem sérios riscos de queda em árvores maduras e potencializam a ocorrência de acidentes (NICLAS, 2002; MATTHECK \& BRELOER, 1997).

O método de avaliação visual de árvores desenvolvido por Mattheck \& Breloer (1994), também conhecido como VTA (visual tree assessment), sugere três diferentes ferramentas para análise interna das árvores: o som de pancadas de martelo na árvore, o penetrômetro e o medidor de fraturas. O VTA representou um grande avanço na detecção de defeitos na madeira, porém existe um grande interesse no desenvolvimento e aplicação de técnicas mais rápidas e precisas de diagnóstico (NICOLOTTI et al., 2003).

A literatura especializada menciona inúmeras técnicas de análise não destrutivas do lenho das árvores, que segundo Nicolotti et al. (2003) seriam mais apropriadamente chamadas de "quase não destrutivas", pois, em muitos casos, sondas, eletrodos e transdutores são inseridos dentro da árvore. Essas técnicas fornecem informações de pequenas porções da madeira, tornando-se, muitas vezes, necessário realizar mais medições em diferentes pontos para conhecer a seção investigada da árvore. A densitometria por raio $X$ é uma técnica que se enquadra nesse perfil, por meio da qual é feita a medição da densidade aparente da madeira a partir de filmes de raio $X$ de amostras finas de madeira, geralmente obtidas por sonda, de seção transversal do tronco (GLOSSARY OF DENDROCHRONOLOGY, 2006).

Recentemente, as técnicas não destrutivas têm sido desenvolvidas por investigação tomográfica. A tomografia permite a reconstrução de seções transversais inteiras da árvore, por meio de informações fornecidas pela passagem de energia através da madeira. Não havia instrumentos que possibilitassem conhecer seções inteiras de árvores com tamanha rapidez e praticidade, revelando a revolução que representa a tomografia para a avaliação da qualidade do interior das árvores. Além disso, sua utilização deixa pouquíssimos sinais. Configura-se, porém, numa técnica em desenvolvimento e, portanto, carente de estudos.

Diferentes tipos de energia podem ser utilizados em tomografia para fornecer informações sobre a madeira, como ondas de ultra-som, campo elétrico e ondas eletromagnéticas, raios y e raios X (NICOLOTTI et al., 2003).

A tomografia de impulso, por sua vez, baseia-se no princípio da cronometragem de ondas mecânicas para reconstrução de seções das árvores (RINNTECH, 2005).

O tomógrafo de impulso é composto por sensores que são fixados em seções transversais da árvore. Tais sensores recebem pancadas de martelo que produzem ondas mecânicas, cujos tempos de percurso entre os sensores são medidos, e suas velocidades 
calculadas pelo software do equipamento. É gerado, então, um gráfico de velocidade das ondas mecânicas, também chamado de imagem tomográfica. A velocidade do impulso dentro da árvore está altamente correlacionada com a densidade do material (RINNTECH, 2005), o que possibilita a comparação entre valores dessas variáveis.

Assim, o presente trabalho visou analisar a confiabilidade das informações fornecidas pela tomografia de impulso para avaliação do interior do lenho de árvores, por meio da comparação entre os dados de velocidade de onda mecânica obtidos pela aplicação do tomógrafo de impulso com os dados de densidade aparente advindos da densitometria de raio $\mathrm{X}$, em amostras coletadas das espécies Eucalyptus saligna Sm. (eucalipto), Schizolobium parahyba (Vell.) Blake (guapuruvu), Caesalpinia echinata Lam. (pau-brasil).

\section{MATERIAL E MÉTODOS}

As amostras de lenho utilizadas no experimento provinham de árvores das espécies Eucalyptus saligna Sm. (eucalipto), Schizolobium parahyba (Vell.) Blake (guapuruvu), Caesalpinia echinata Lam. (pau-brasil). Foi coletado um torete (com no mínimo $20 \mathrm{~cm}$ de altura) da região basal de um exemplar de cada espécie tombado no Parque da ESALQUSP durante mini-ciclone ocorrido em março de 2006. As medições foram realizadas no Laboratório de Silvicultura Urbana e no Laboratório de Anatomia da Madeira do Departamento de Ciências Florestais da Escola Superior de Agricultura Luiz de Queiroz (ESALQ-USP).

Em seção transversal à altura média dos toretes foram fixados 12 pregos eqüidistantes, atravessando as regiões da casca/alburno (até $1 \mathrm{~cm}$ ), para sustentação dos sensores do tomógrafo de impulso Arbotom. Cada sensor recebeu 6 pancadas de martelo para produção de ondas mecânicas. As velocidades das ondas - no lenho - entre os sensores foram calculadas pelo software do equipamento, produzindo o respectivo gráfico de velocidade de onda da seção transversal (imagem tomográfica).

Foram cortados discos de $5 \mathrm{~cm}$ de espessura, que incluíam a seção transversal de produção das imagens tomográficas. Nos discos, foram demarcadas e cortadas amostras diametrais com $1,5 \mathrm{~cm}$ de largura em serra de disco e, destas, cortadas amostras transversais em equipamento de dupla-serra, com $2 \mathrm{~mm}$ de espessura. Acondicionadas em sala a $20^{\circ} \mathrm{C}$ e $50 \%$ de umidade relativa por 12 horas, as amostras transversais foram homogeneizadas a $12 \%$ de umidade relativa. Posteriormente foram radiografadas com aparelho Hewlett Packard Faxitron 43850 e reveladas (BANZATTO \& TOMAZELLO FILHO, 1998). Os filmes radiográficos foram digitalizados e os valores pontuais, bem como os 
respectivos gráficos dos perfis de densidade aparente das amostras diametrais de lenho foram obtidos pela aplicação dos softwares CRAD e CERD.

Para comparar os dados obtidos pela densitometria de raio $\mathrm{X}$ e pela tomografia de impulso, selecionou-se a faixa da imagem tomográfica na qual estaria contida a amostra diametral da seção transversal analisada pela densitometria de raio $X$. Dessa faixa, percorreu-se uma linha de pixels. As cores dos pixels da linha percorrida foram reconhecidas com o auxílio do software Adobe Photoshop, sendo associado um valor de velocidade de onda a cada cor. Coonfeccionou-se dos gráficos de perfil de velocidade de onda mecânica das amostras de lenho, a semelhança da densitometria.

Também, produziu-se o diagrama de dispersão das médias de velocidade de onda mecânica vs. médias de densidade aparente, de faixas de $3 \mathrm{~cm}$ dos gráficos de perfil das amostras, acompanhado da respectiva equação de regressão polinomial de $2^{\circ} \mathrm{grau}$, e foi aplicado Teste t para comparação entre as médias.

\section{RESULTADOS E DISCUSSÃO}

A aplicação do tomógrafo de impulso nas amostras de lenho de Eucalyptus saligna Sm. (eucalipto), Schizolobium parahyba (Vell.) Blake (guapuruvu), Caesalpinia echinata Lam. (pau-brasil) gerou as respectivas imagens tomográficas apresentadas nas Figuras 1, 2 e 3. Também estão demarcadas nas imagens tomográficas as faixas que continham as amostras diametrais analisadas pela densitometria de raio $\mathrm{X}$.

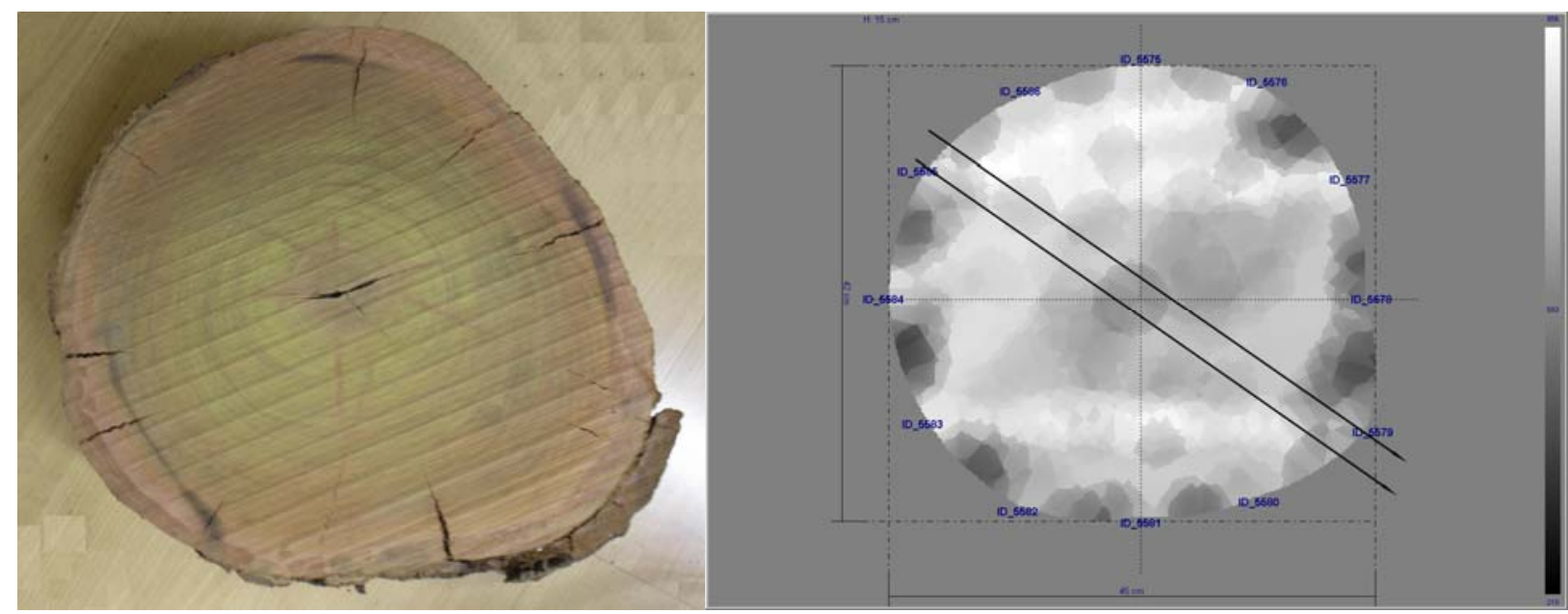

Figura 1- Eucalipto: foto da seção transversal do torete e respectiva imagem tomográfica com demarcação da faixa correspondente à amostra diametral analisada pela densitometria de raio $X$. 


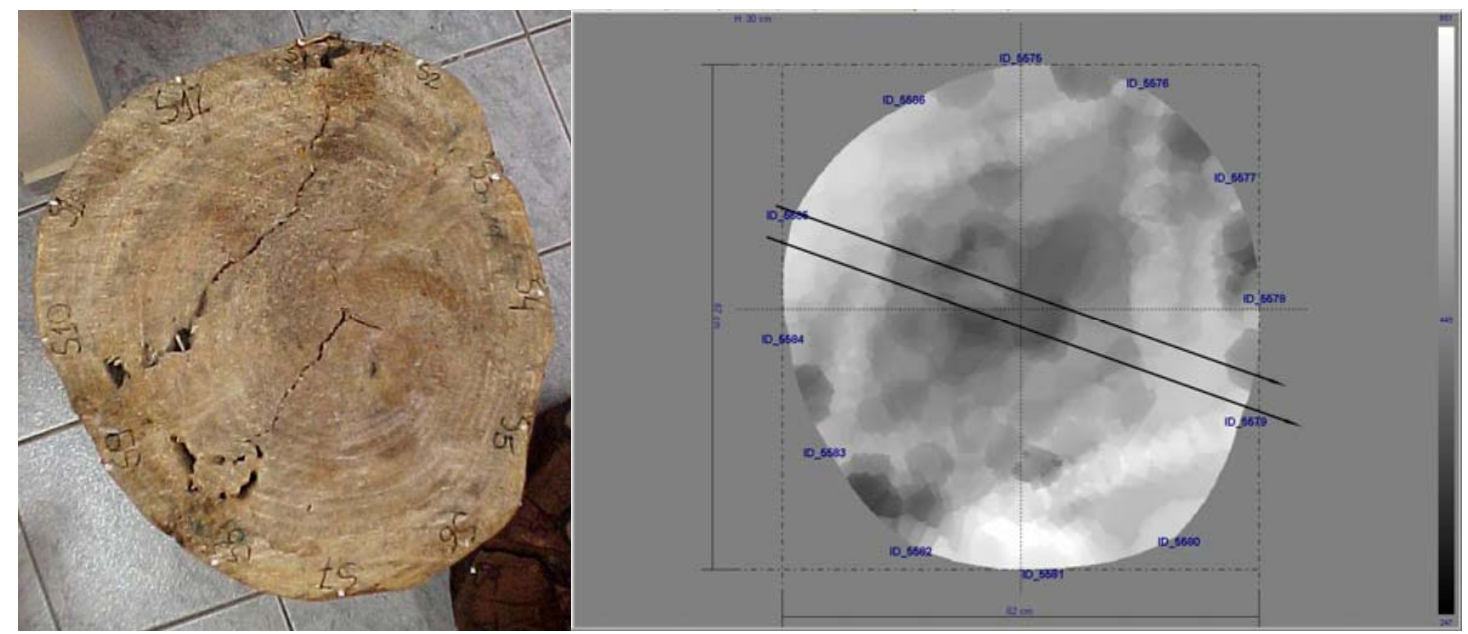

Figura 2- Guapuruvu: foto da seção transversal do torete e respectiva imagem tomográfica com demarcação da faixa correspondente à amostra diametral analisada pela densitometria de raio $X$.

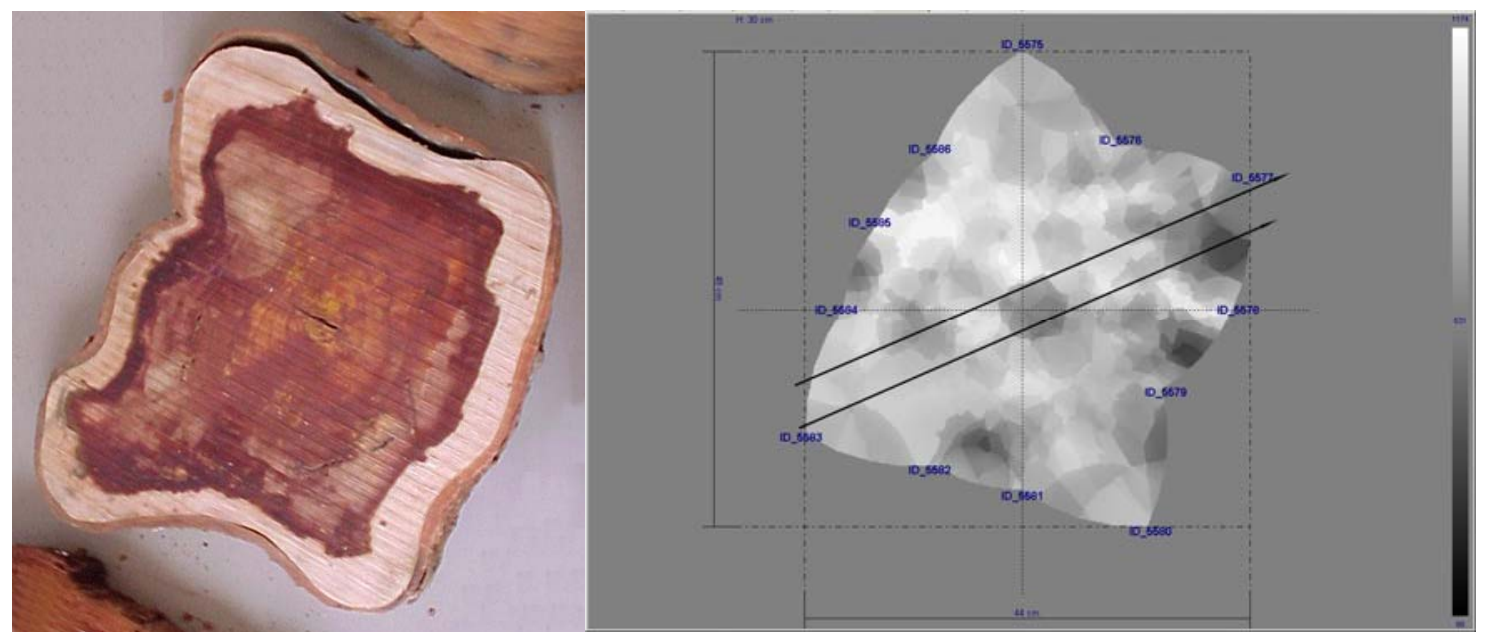

Figura 3- Pau-brasil: foto da seção transversal do torete e respectiva imagem tomográfica com demarcação da faixa correspondente à amostra diametral analisada pela densitometria de raio $X$.

Os gráficos de perfil de densidade aparente (densitometria de raio $\mathrm{X}$ ) seguidos pelos gráficos de perfil de velocidade de onda mecânica (tomografia de impulso) das amostras são mostrados nas Figuras 4, 5 e 6. Pela observação dos pares de gráficos, nota-se que existem semelhanças entre as suas curvas, tanto no primeiro raio (representado pelos valores negativos de posição) como no segundo raio (valores positivos de posição). 

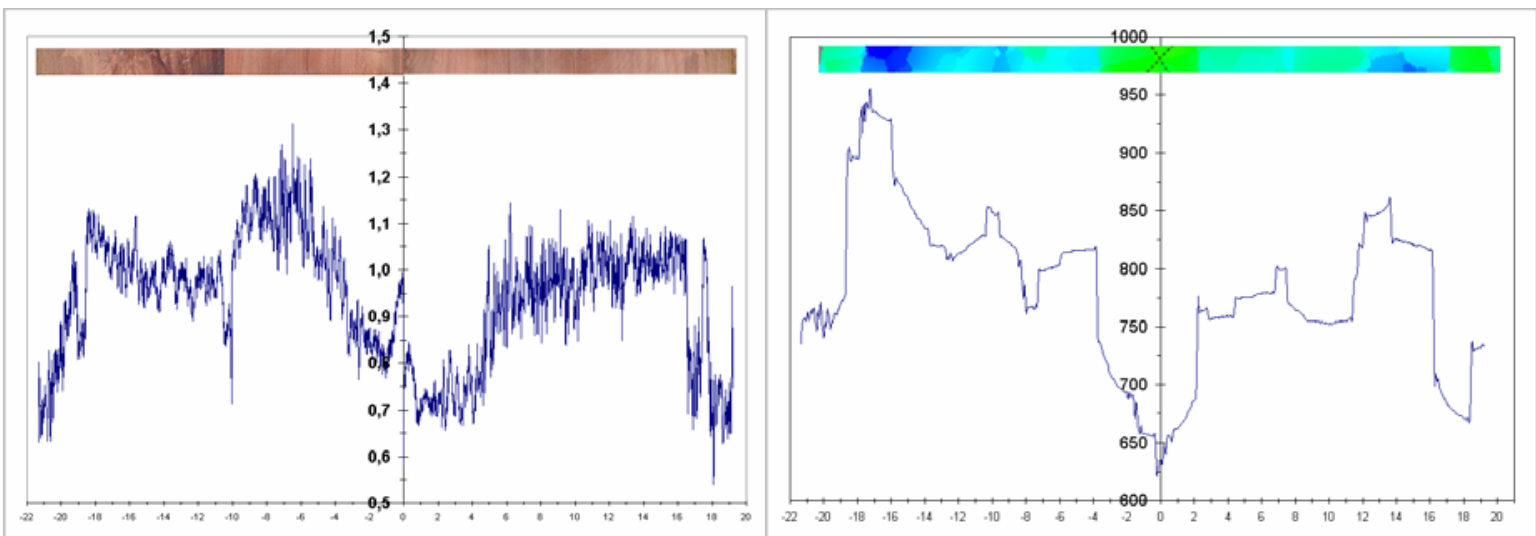

Figura 4 - Eucalipto: gráfico de perfil de densidade aparente e gráfico de perfil de velocidade de onda mecânica.
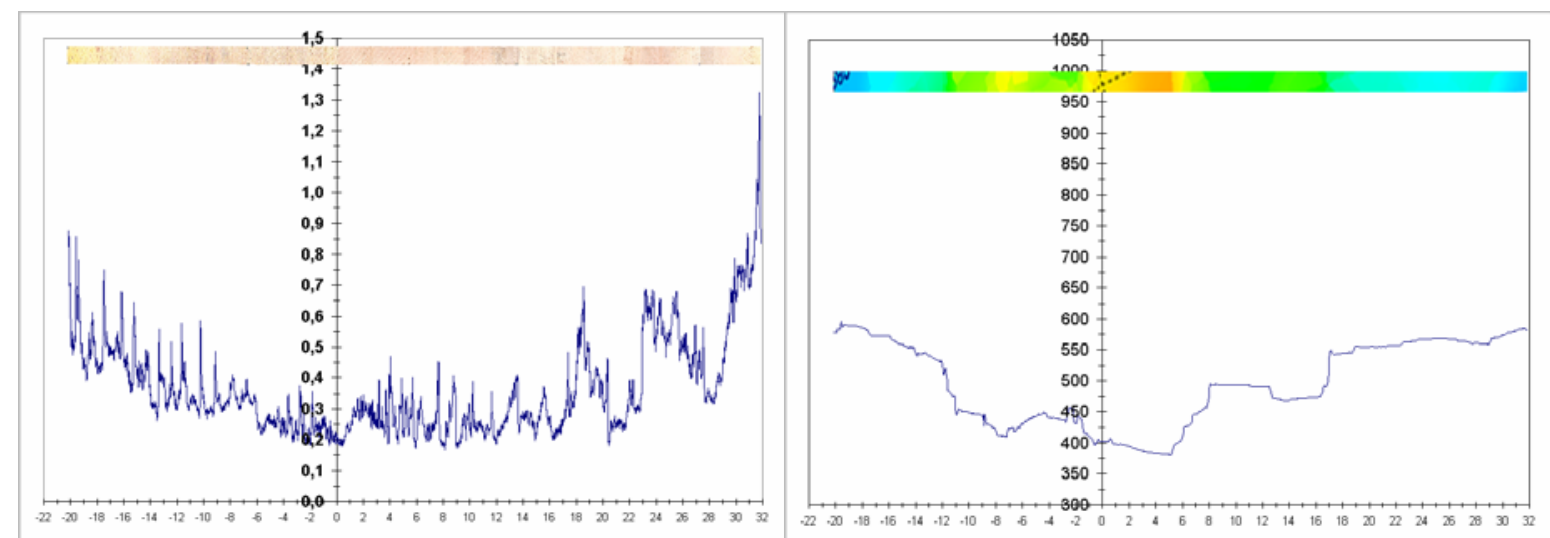

Figura 5 - Guapuruvu: gráfico de perfil de densidade aparente e gráfico de perfil de velocidade de onda mecânica.
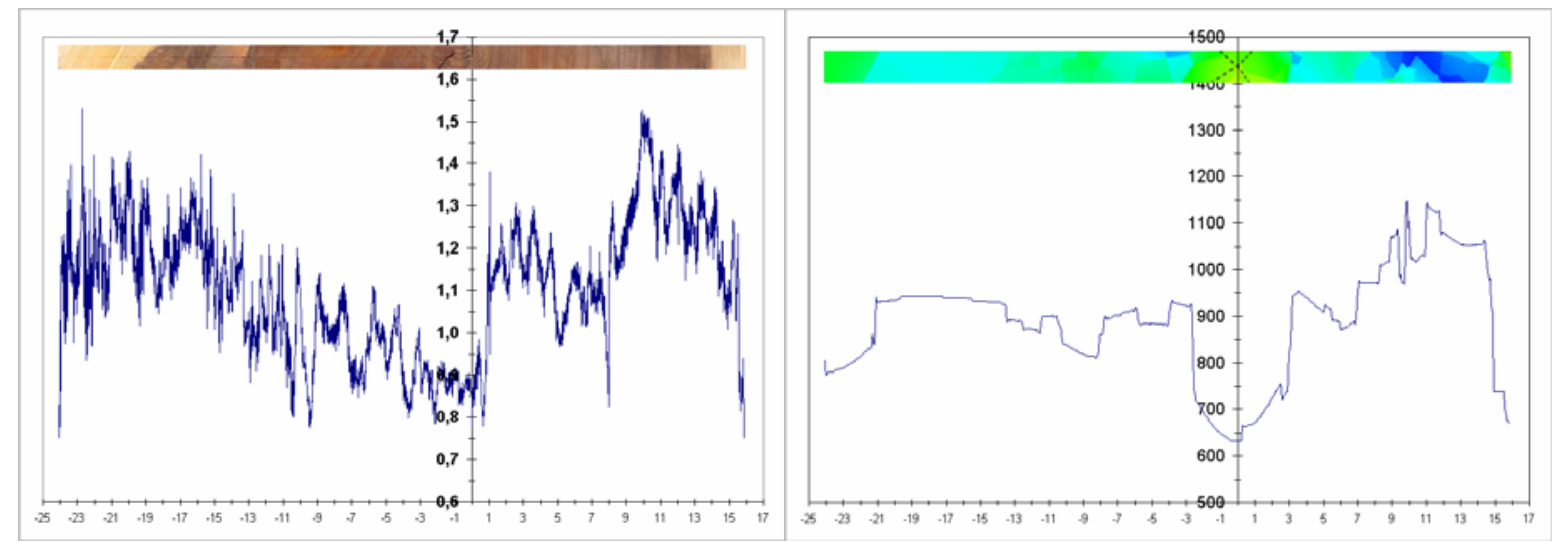

Figura 6 - Pau-Brasil: gráfico de perfil de densidade aparente e gráfico de perfil de velocidade de onda mecânica.

O diagrama de dispersão e a equação de regressão polinomial de $2^{\circ}$ grau das médias de velocidade de onda vs. médias de densidade aparente para cada espécie (Figura $7,8,9)$ permitem analisar quanto os dados obtidos pela tomografia de impulso são 
semelhantes aos obtidos pela densitometria de raio $\mathrm{X}$. Os asteriscos indicam que o valor do coeficiente de determinação $\left(R^{2}\right)$ foi significativo pelo teste de Tukey a $1 \%$, portanto as médias de densidade aparente e de velocidade de onda obtidas ao longo do perfil do lenho são semelhantes para todas as amostras.

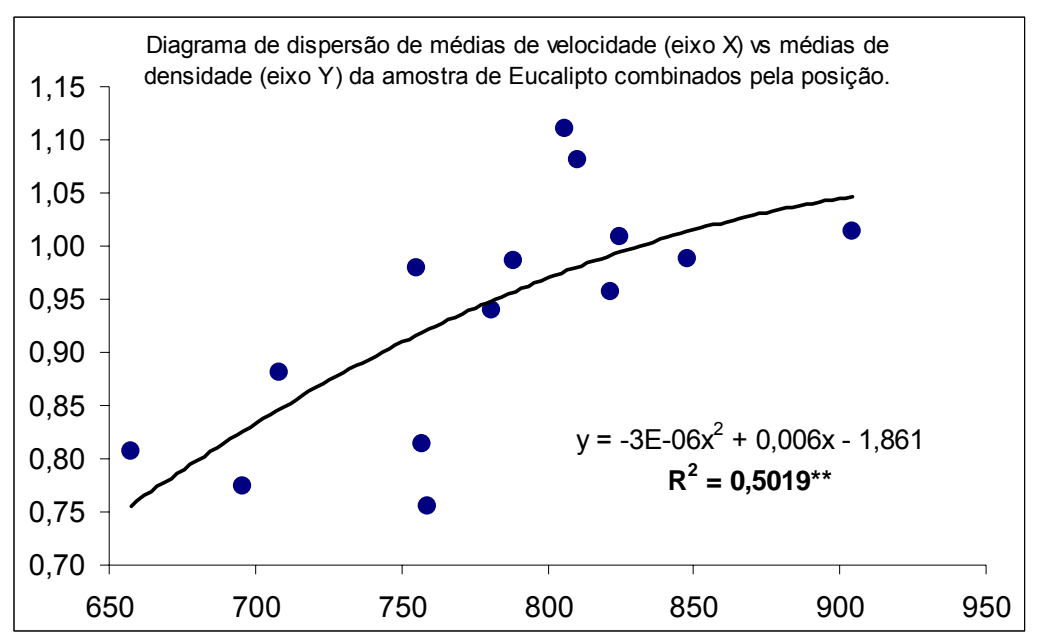

\begin{tabular}{|c|c|c|}
\hline \multirow[b]{2}{*}{$\begin{array}{c}\text { Faixas } \\
\text { (cm) }\end{array}$} & \multicolumn{2}{|r|}{ Densidad } \\
\hline & $\begin{array}{c}\text { Velocidade } \\
\qquad(\mathrm{m} / \mathrm{s})\end{array}$ & $\begin{array}{c}\text { Ap. } \\
\left(\mathrm{g} / \mathrm{cm}^{3}\right)\end{array}$ \\
\hline $\begin{array}{c}-22--19 \\
-19--16 \\
-16--13 \\
-13--10 \\
-10--7 \\
-7--4 \\
-4--1 \\
-1-2 \\
2-5 \\
5-8 \\
8-11 \\
11-14 \\
14-17 \\
17-20 \\
\end{array}$ & $\begin{array}{l}757,15 \\
904,20 \\
848,10 \\
821,73 \\
805,78 \\
810,14 \\
708,49 \\
657,43 \\
758,95 \\
781,17 \\
755,36 \\
824,95 \\
788,63 \\
696,02 \\
\end{array}$ & $\begin{array}{l}0,81 \\
1,01 \\
0,99 \\
0,96 \\
1,11 \\
1,08 \\
0,88 \\
0,81 \\
0,76 \\
0,94 \\
0,98 \\
1,01 \\
0,99 \\
0,77 \\
\end{array}$ \\
\hline
\end{tabular}

Figura 7 - Eucalipto: Diagrama de dispersão de médias de velocidade (eixo X) vs. médias de densidade (eixo y) por faixa de posição e tabela de dados de origem. Os asteriscos indicam $\mathrm{R}^{2}$ significativo a $1 \%$ pelo teste $t$. 


\begin{tabular}{|c|c|c|}
\hline \multirow[b]{2}{*}{$\begin{array}{c}\text { Faixas } \\
(\mathrm{cm})\end{array}$} & \multicolumn{2}{|r|}{ Densidade } \\
\hline & $\begin{array}{c}\text { Velocidade } \\
\qquad(\mathrm{m} / \mathrm{s})\end{array}$ & $\begin{array}{c}\text { Ap. } \\
\left(\mathrm{g} / \mathrm{cm}^{3}\right)\end{array}$ \\
\hline$-21--18$ & 586,59 & 0,54 \\
\hline$-18--15$ & 571,51 & 0,48 \\
\hline -15--12 & 544,64 & 0,37 \\
\hline$-12--9$ & 463,22 & 0,34 \\
\hline$-9--6$ & 422,32 & 0,33 \\
\hline$-6--3$ & 440,66 & 0,25 \\
\hline$-3-0$ & 425,19 & 0,23 \\
\hline $0-3$ & 396,69 & 0,26 \\
\hline $3-6$ & 387,99 & 0,27 \\
\hline $6-9$ & 460,60 & 0,26 \\
\hline $9-12$ & 492,03 & 0,24 \\
\hline $12-15$ & 474,54 & 0,28 \\
\hline $15-18$ & 501,43 & 0,28 \\
\hline $18-21$ & 552,34 & 0,39 \\
\hline $21-24$ & 560,56 & 0,42 \\
\hline $24-27$ & 566,94 & 0,54 \\
\hline $27-30$ & 563,94 & 0,46 \\
\hline $30-33$ & 579,95 & 0,81 \\
\hline
\end{tabular}

Figura 8 - Guapuruvu: Diagrama de dispersão de médias de velocidade (eixo X) vs. médias de densidade (eixo y) por faixa de posição e tabela de dados de origem. Os asteriscos indicam $\mathrm{R}^{2}$ significativo a $1 \%$ pelo teste $\mathrm{t}$.

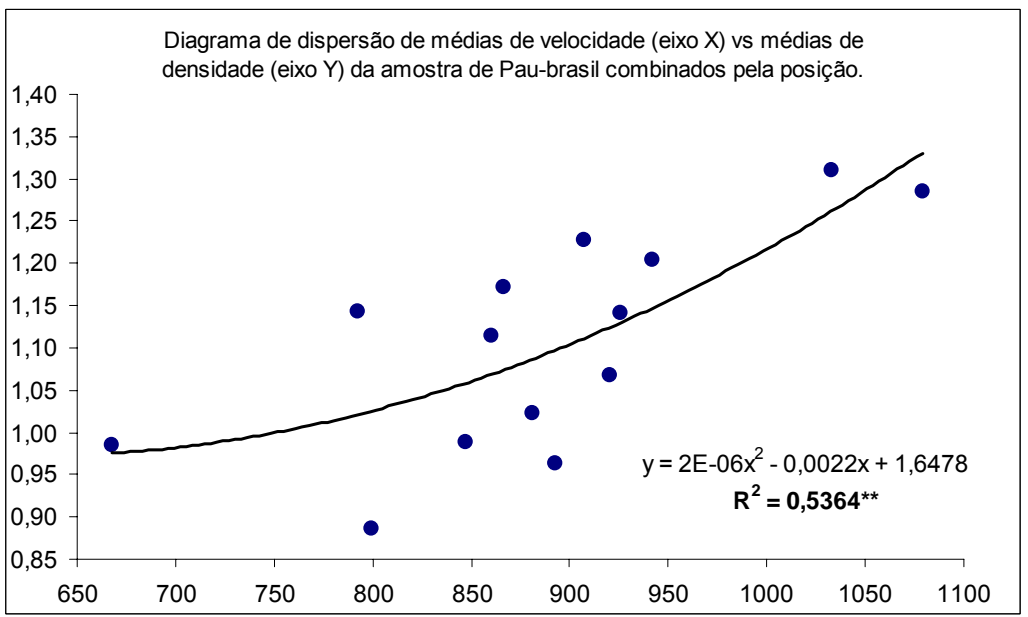

\begin{tabular}{ccc}
$\begin{array}{c}\text { Faixas } \\
(\mathrm{cm})\end{array}$ & $\begin{array}{c}\text { Velocidade } \\
(\mathrm{m} / \mathrm{s})\end{array}$ & $\begin{array}{c}\text { Ap. } \\
\left(\mathrm{g} / \mathrm{cm}^{3}\right)\end{array}$ \\
\hline$-25--22$ & 792,38 & 1,14 \\
$-22--19$ & 907,44 & 1,23 \\
$-19--16$ & 941,78 & 1,20 \\
$-16--13$ & 925,70 & 1,14 \\
$-13--10$ & 881,28 & 1,02 \\
$-10--7$ & 846,99 & 0,99 \\
$-7-4$ & 893,06 & 0,96 \\
$-4--1$ & 799,25 & 0,89 \\
$-1-2$ & 667,88 & 0,99 \\
$2-5$ & 866,74 & 1,17 \\
$5-8$ & 920,79 & 1,07 \\
$8-11$ & 1032,81 & 1,31 \\
$11-14$ & 1078,86 & 1,29 \\
$14-17$ & 860,27 & 1,11 \\
\hline
\end{tabular}

Figura 9 - Pau-Brasil: Diagrama de dispersão de médias de velocidade (eixo X) vs. médias de densidade (eixo y) por faixa de posição e tabela de dados de origem. Os asteriscos indicam $\mathrm{R}^{2}$ significativo a $1 \%$ pelo teste $\mathrm{t}$. 
Ocorreram variações entre os pares de gráficos de perfil, provavelmente devido ao método usado para obtenção dos valores de velocidade de onda mecânica - em uma linha de pixels da imagem tomográfica - à semelhança da densitometria de raio $X$. Tal método é específico da densitometria (apenas uma amostra diametral), enquanto a tomografia de impulso reproduz regiões na totalidade da secção transversal. O tomógrafo de impulso detecta regiões. $O$ raio $X$ possibilita a obtenção de valores precisos em uma amostra diametral, sendo inviável sua utilização para avaliar áreas completas de uma seção do tronco.

\section{CONCLUSÃO}

Por meio dos métodos usados e resultados encontrados é possível afirmar que:

- A tomografia de impulso produziu informações similares às produzidas pela densitometria de raio $X$, demonstrando ser eficaz para obtenção de dados sobre a integridade das amostras de lenho estudadas das espécies: Eucalyptus saligna Sm. (eucalipto), Schizolobium parahyba (Vell.) Blake (guapuruvu) e Caesalpinia echinata Lam. (pau-brasil);

- A tomografia de impulso pode ser recomendada para avaliação do interior do lenho de árvores maduras e conseqüentemente auxiliar na avaliação do risco de queda;

- Mais experimentações devem ser feitas para relacionar áreas internas do lenho com valores de densidade e risco de queda de árvores maduras.

As conseqüências deste e demais estudos futuros serão valiosas para as avaliações técnicas de árvores em áreas urbanas. Atualmente no Brasil, as avaliações técnicas quali-quantitativas são apenas visuais em inventários e cadastros (SILVA FILHO et al., 2002).

\section{AGRADECIMENTOS}

Os autores agradecem a FAPESP - Fundação de Amparo à Pesquisa do Estado de São Paulo pelos recursos disponibilizados para realização desta pesquisa, por meio da linha de trabalhos em Políticas Públicas.

\section{REFERÊNCIAS BIBLIOGRÁFICAS}

GLOSSARY OF DENDROCHRONOLOGY. Densitometria de raio-X. Disponível em $<$ http://www01.wsl.ch/glossary/FMPro?-db=dendroglossary.fp5\&-lay=web\&-format=detailspo.html\&ID_Nr=350\&-Find>. Acesso em: 03 dez. 2006. 
MATTHECK, C.; BRELOER, $H$. The body language of trees: a handbook for failure analysis. London: Her Majesty s Stationery Office, 260p, 1997.

MATTHECK, C.; BRELOER, H. Field guide for visual tree assessment (VTA). Journal of Arboriculture, 18:1-23, 1994.

NICOLOTTI, G.; SOCCO, L. V.; MARTINIS, R.; GODIO, A.; SAMBUELLI, L. Application and comparison of three tomographic tecniques for detection of decay in trees. Journal of Arboriculture 29(2): 66-78, 2003.

OLIVEIRA, J. T. da S.; S, J. de C. Variação radial da retratibilidade e densidade básica da madeira de Eucalyptus saligna Sm.. Rev. Árvore, Jun 2003, vol.27, no.3, p.381-385.

RINNTECH. User Manual - Arbotom 3-D Tree Impulse Tomograph, version 1.59 for Microsoft Windows 98, 2000, XP. 42p., 2005.

SILVA FILHO, D.F., PIZETTA, P.U. C., ALMEIDA, J.B.S.A. de et al. Relational database for inventory system evaluation and management of urban street trees. Rev. Árvore, Out. 2002, vol.26, no.5, p.629-642. 